\title{
When GWAS meets the Connectivity Map: drug repositioning for seven psychiatric disorders
}

Hon-Cheong So ${ }^{1,2^{*}}$, Carlos K.L. Chau ${ }^{1}$, Wan-To $\mathrm{Chiu}^{3}$, Kin-Sang $\mathrm{Ho}^{3}$, Cho-Pong $\mathrm{Lo}^{3}$, Stephanie Ho-Yue Yim ${ }^{4}$ and Pak C. Sham ${ }^{5,6,7,8}$

${ }^{1}$ School of Biomedical Sciences, The Chinese University of Hong Kong, Shatin, Hong Kong

${ }^{2}$ KIZ-CUHK Joint Laboratory of Bioresources and Molecular Research of Common Diseases, Kunming Zoology Institute of Zoology and The Chinese University of Hong Kong

${ }^{3}$ Faculty of Medicine, The Chinese University of Hong Kong, Shatin, Hong Kong

${ }^{4}$ Univeristy of Exeter Medical School, United Kingdom

${ }^{5}$ Department of Psychiatry, University of Hong Kong, Pokfulam, Hong Kong

${ }^{6}$ Centre for Genomic Sciences, University of Hong Kong, Pokfulam, Hong Kong

${ }^{7}$ State Key Laboratory for Cognitive and Brain Sciences, University of Hong Kong, Pokfulam, Hong Kong

${ }^{8}$ Centre for Reproduction, Development and Growth, University of Hong Kong, Pokfulam, Hong Kong

*Correspondence to: Hon-Cheong So, School of Biomedical Sciences, The Chinese University of Hong Kong, Shatin, Hong Kong

Email: hcso@cuhk.edu.hk 


\begin{abstract}
Our knowledge of disease genetics has advanced rapidly during the past decade, with the advent of high-throughput genotyping technologies such as genome-wide association studies (GWAS). However, few methodologies were developed and systemic studies performed to identify novel drug candidates utilizing GWAS data. In this study we focus on drug repositioning, which is a cost-effective approach to shorten the developmental process of new therapies. We proposed a novel framework of drug repositioning by comparing GWAS-imputed transcriptome with drug expression profiles from the Connectivity Map. The approach was applied to 7 psychiatric disorders. We discovered a number of novel repositioning candidates, many of which are supported by preclinical or clinical evidence. We found that the predicted drugs are significantly enriched for known psychiatric medications, or therapies considered in clinical trials. For example, drugs repurposed for schizophrenia are strongly enriched for antipsychotics $(p=4.69 \mathrm{E}-06)$, while those repurposed for bipolar disorder are enriched for antipsychotics $(p=2.26 \mathrm{E}-07)$ and antidepressants $(p=1.17 \mathrm{E}-05)$. These findings provide support to the usefulness of GWAS signals in guiding drug discoveries and the validity of our approach in drug repositioning. We also present manually curated lists of top repositioning candidates for each disorder, which we believe will serve as a useful resource for researchers.
\end{abstract}




\section{INTRODUCTION}

The last decade has witnessed a rapid growth of genotyping technologies and genome-wide association studies (GWAS) have played in major role in unraveling the genetic bases of complex diseases. According to the latest GWAS catalog (www.ebi.ac.uk/gwas/, accessed $22^{\text {nd }}$ Dec 2016), more than 2600 GWAS studies have been performed to date. It is worth noting that many biobanks are also collecting genomic data, and the private genetics company 23andMe has genotyped more than a million customers (mediacenter.23andme.com/fact-sheet/). It is clearly of great clinical and public interest to translate these findings into treatment for diseases. Nevertheless, compared to the large literature of GWAS studies, relatively few methodologies have been developed and systematic studies performed to identify novel drug candidates by using GWAS data.

Psychiatric disorders carry significant burden on health globally ${ }^{1}$ and the current treatment strategies are far from perfect. Despite the heavy health burden and the increased awareness of mental health in many places, drug discovery in the field have largely been stagnant ${ }^{2}$. As argued by Hyman, the basic mechanisms of most antidepressants and antipsychotics, the most widely used drugs used in psychiatry, are relatively similar to their prototypes imipramine and chlorpromazine discovered in the $1950 \mathrm{~s}^{2}$. On the other hand, in recent years GWAS studies have greatly advanced our knowledge of the genetic bases of many psychiatric disorders. Taking advantage of these developments, we proposed a new framework for identifying drug candidates based on GWAS results, and applied the method to a variety of psychiatric disorders.

Here we focus on drug repositioning, that is, finding new indications for existing drugs. As conventional drug development is an expensive and lengthy process, repositioning serves as a useful strategy to hasten the development cycle $^{3}$. It is worth noting that while we use the term 
"drug repositioning" throughout this paper, the method is also applicable to any chemicals with known gene expression profiles.

A few previous studies have investigated the use of GWAS data in drug repositioning. The most intuitive approach is to study whether the top genes identified in GWAS can serve as drug targets. Sanseau et al. ${ }^{4}$ searched for top susceptibility genes (with $p<1 \mathrm{e}-7$ ) from the GWAS catalog and matched the results against the drug targets listed in the Pharmaprojects database, a commercial resource with information on global drug pipelines. In addition, they proposed a repositioning approach by identifying "mismatches" between drug indications and the original GWAS traits. In another study ${ }^{5}$, Cao et al. considered interacting partners of GWAS hits to discover new drug targets. Lencz and Malhotra ${ }^{6}$ compared schizophrenia GWAS results with drug target genes, and prioritized potential new drug targets for the disease. Another recent study investigated whether evidence from human genetic studies are useful to drug development in general. The authors found that the proportion of drugs with support from GWAS increased along the development pipeline ${ }^{7}$.

While the approach of finding overlap between top GWAS hits and known drug target genes is useful, it has a number of limitations. Firstly, many of the top GWAS genes may not be easily targeted by a drug. Cao and Moult ${ }^{5}$ studied 856 drug target genes from DrugBank and found only 20 genes that were discovered in GWAS of the corresponding disease. While this number is likely to improve with increasing sample sizes, there will remain a number of GWAS hits which are not directly "druggable". In addition, many of the GWAS top SNPs are within non-coding regions ${ }^{8}$ and do not encode for a drug target protein. The above approach might also miss "multi-target" drugs, a paradigm that has attracted increasing attention in recent years. It is argued that as complex diseases (like most psychiatric disorders) involve the interplay of multiple genetic and environmental factors, they may be more easily managed by 
modulating multiple instead of single targets ${ }^{9}$. Lastly, as previous studies mainly focused mainly on the most significant hits, they ignored the contribution of genetic variants with smaller effect sizes. As shown in polygenic score analyses of many complex traits, often variants achieving lower significance levels also contribute to disease risks ${ }^{10}$.

With the aforementioned limitations in mind, we developed a new strategy for drug repositioning by imputing gene expression profile from GWAS summary statistics and comparing it with drug-induced expression changes. Analysis of the transcriptome of drugs versus diseases is an established approach for drug repositioning and has previously been successfully applied for complex diseases ${ }^{11,12}$. For example, by examining drugs in the Connectivity Map (Cmap) ${ }^{13}$ which showed opposite patterns of expression to diseases, Sirota et al. ${ }^{11}$ derived candidates for repositioning and experimentally validated a prediction cimetidine for the treatment of lung adenocarcinoma. With a similar method, Dudley et al. ${ }^{12}$ identified topiramate as a novel treatment for inflammatory bowel disease and validated it in an animal model. Other studies (e.g. $\left.{ }^{14-16}\right)$ also showed potential of this approach in repositioning.

Built on this repositioning strategy, we proposed a new approach by using imputed transcriptome from GWAS instead of expression data from microarray or RNA-sequencing studies. This approach has several advantages. Firstly, patients from expression studies are often medicated. This is particularly relevant for studies in neuro-psychiatry, as brain tissues can only come from post-mortem samples of patients, who were often on psychiatric medications ${ }^{17}$. History of medications might confound our results as our aim is to compare the expression patterns of disease and those of drugs. Imputed transcriptome on the other hand is not altered by medications or other environmental confounders. Secondly, current GWAS samples are usually orders of magnitude larger than expression studies (often $10^{4}$ or more), 
and GWAS summary statistics are widely available. In addition, for many diseases such as psychiatric disorders, the tissues of interest are not easily accessible. On the other hand, as we shall explain below, expression profiles can be readily imputed for over 90 tissues from GWAS data using appropriate statistical models.

\section{METHODS}

\section{Imputation of expression profile from GWAS data}

Recently methods have been developed to impute expression from GWAS variants ${ }^{18-20}$. The main idea is to build a statistical model to predict expression levels from SNPs in a reference transcriptome dataset, and the prediction model can be applied to new genotype data. This approach estimates the component of gene expression that is contributed by (germline) genetic variations. The program PrediXcan ${ }^{18}$ was developed for this purpose based on models built from the Genotype-Tissue Expression (GTEx) project ${ }^{21}$ and the Depression Genes and Networks (DGN) cohort ${ }^{22}$. As most individual genotype data are not publicly available due to privacy concerns, we applied a recently developed algorithm called MetaXcan which allows imputation of expression $z$-scores (i.e. $z$-statistic derived from comparing gene expression in cases versus controls) based on summary statistics alone. The method was shown to be in excellent concordance with predictions made from raw genotype data ${ }^{19}$. Assuming that a set of SNPs $\left(\mathrm{SNP}_{1}, \mathrm{SNP}_{2} \ldots, \mathrm{SNP}_{k}\right)$ contribute to the expression of gene $g$, the following formula can be used to compute the expression $z$-scores for the gene:

$z_{g} \approx \sum_{i=1}^{k} w_{g i} \frac{\hat{\sigma}_{i}}{\hat{\sigma}_{g}} \frac{\hat{\beta}_{i}}{\operatorname{SE}\left(\hat{\beta}_{i}\right)}$

where $w_{g i}$ is the weight given to $\mathrm{SNP}_{i}$ for predicting the expression level of gene $\mathrm{g}, \hat{\sigma}_{i}$ and $\hat{\sigma}_{g}$ denote the estimated variance of $\mathrm{SNP}_{i}$ and gene $g$ respectively (estimated from a reference genotype dataset), and $\hat{\beta}_{i} / \operatorname{SE}\left(\hat{\beta}_{i}\right)$ denotes the summary z-statistic of $\mathrm{SNP}_{i}$ of the disease 
trait. The weights of SNPs $w_{g i}$ were computed from reference datasets of expression quantitative trait loci (eQTL) studies. We downloaded pre-computed weights derived from an elastic net prediction model with GTEx as the reference transcriptome dataset, provided by the authors of MetaXcan. It is worth noting that the above methodology is similar to another transcriptome imputation algorithm TWAS $^{20}$, but a different prediction method (based on a mixed model) is employed in TWAS. We used MetaXcan in this study as the program readily allows transcriptome imputation for a much larger variety of tissues.

\section{Transcriptome imputation of 7 psychiatric disorders}

GWAS summary statistics were obtained from the Psychiatric Genomics Consortium (PGC) website (www.med.unc.edu/pgc/results-and-downloads). We downloaded eight sets of GWAS summary statistics corresponding to seven psychiatric disorders, including schizophrenia $(\mathrm{SCZ})^{23}$, major depressive disorder (MDD) ${ }^{24,25}$, bipolar disorder $(\mathrm{BD})^{26}$, Alzheimer's disease $(\mathrm{AD})^{27}$, anxiety disorders $(\mathrm{ANX})^{28}$, autistic spectrum disorders $(\mathrm{ASD})^{29}$ and attention deficit hyperactivity disorder (ADHD) ${ }^{30}$. We employed two different sets of summary statistics for MDD; the first set was from the PGC group ${ }^{24}$, while the other was from the CONVERGE study which recruited more severe MDD cases from Chinese women only ${ }^{25}$. Details of individual studies are described in the respective references. Transcriptome of each disease was imputed for ten brain regions included in GTEx, using default parameters in MetaXcan.

\section{Drug-induced expression profiles}

Drug-induced expression profiles were derived from the Cmap database, a resource of genome-wide expression profile of cultured cell lines treated with 1309 different drugs or small molecules ${ }^{13}$. We downloaded raw expression data from Cmap, and performed normalization with the MAS5 algorithm. Expression levels of genes represented on more than 
one probe sets were averaged. Differential expression between treated cell lines and controls was tested using the limma package ${ }^{31}$. We performed analyses on each combinations of drug and cell line, with a total of 3478 instances. Statistical analyses were performed in R3.2.1 with the assistance of the R package "longevityTools".

\section{Comparison of gene expression profiles of drugs versus diseases}

Next we compared the expression profiles (in z-scores) of drugs versus those of diseases. The original study on Cmap employed Kolmogorov-Smirnov (KS) tests ${ }^{13}$ to compare two expression patterns. In brief, the aim of the KS test is to evaluate whether a set of disease-related genes are ranked higher or lower than expected in a list of genes sorted by their drug-induced expression levels. The KS test was performed separately for upregulated and downregulated disease genes. For drug repositioning, we study whether there is an enrichment of genes that are upregulated for disease but downregulated on drug treatment, and vice versa. We adopted the same formulae described in original Cmap paper to calculate the "connectivity scores".

Reversed patterns of expression can also be tested by Spearman or Pearson correlations. Yet another approach is to use only the $K$ most up- or down-regulated genes in computing the correlations $^{32}$. In this study we employed all five methods (i.e. KS test, Spearman correlation with all or the most differentially expressed genes, Pearson correlation with all or the most differentially expressed genes) in our analyses and computed the average ranks. As there are no consensus methods to define $K$, we also set different values of $K(50,100,250,500)$ and averaged the results for each method. Drugs were then ranked in ascending order of their connectivity scores or correlation results (i.e. the most negative correlation or connectivity score ranked first).

To assess the significance of the ranks, we performed permutation tests by shuffling the 
disease expression z-scores and comparing it to drug transcriptomic profiles. One hundred permutations were performed for each drug-disease pair, and the distribution of ranks under the null was combined across all drug-disease pairs (such that the null distribution was derived from 347800 ranks under $\mathrm{H}_{0}$ ).

\section{Manual curations of the top repositioning candidates}

To assess the drug candidates found in our drug repositioning algorithm, we performed manual curations of the top 15 drugs (representing the top $\sim 0.45 \%$ of all instances) identified for each disorder and brain region. Literature search was performed to look for evidence of therapeutic potential of the identified drug candidates.

\section{Tests for enrichment of known indicated drugs or drugs in clinical trial}

In addition to manually inspecting the top candidates, in order to validate our approach, we also tested for an enrichment of drugs that are (1) indicated for each disorder; or (2) included in clinical trials. The enrichment tests are similar in principle to gene-set analyses, but with gene-sets replaced by drug-sets. We employed two types of tests for enrichment. In the first approach, we tested whether a known drug-set, such as antipsychotics or antidepressants, were ranked significantly higher than by chance; this approach is also known as a "self-contained" test. In the second method, we compared medications in the drug-set against those outside the set, and tested whether the former group was ranked significantly higher. This is also known as a "competitive" test ${ }^{33,34}$. Details of the statistical methods are described in Supplementary Text.

We considered three sources of drug-sets in our analyses. The first set comes from the Anatomical Therapeutic Classification (ATC) drugs downloaded from KEGG. The ATC is an established system for the classification of medications and we extracted three groups of 
drugs: (1) all psychiatric drugs (coded "N05" or "N06"); (2) antipsychotics (coded "N05A"); (3) antidepressants and anxiolytics (coded "N05B" or "N06A"). We grouped antidepressants and anxiolytics together in our analyses as many anti-depressants are indicated for anxiety disorders, and vice versa, anxiolytics are frequently prescribed to depressive patients clinically $^{35}$. We did not specifically include drugs for dementia or psychostimulants as they are relatively few in number. Note that the ATC does not classify drugs specifically for some disorders, such as BD, autism and ADHD.

The second source is from Wei et al. ${ }^{36}$ who complied a MEDication Indication resource (MEDI) from four public medication resources, including RxNorm, Side Effect Resource 2 (SIDER2), Wikipedia and MedlinePlus. A random subset of the extracted indications was also reviewed by physicians. We used the MEDI high-precision subset (MEDI-HPS) which only include drug indications found in RxNorm or in at least 2 out of 3 other sources, with an estimated precision of $92 \%$.

As the aforementioned sources only include known drug indications, we also considered a wider set of drugs that are included for clinical trials on https://clinicaltrials.gov. These drugs represent promising candidates that are often supported by preclinical or human studies. We downloaded a precompiled list of these drugs (created in May 2016) from https://doi.org/10.15363/thinklab.d212.

For each disorder, we compiled a list of repositioning candidates using the imputed transcriptome profile of each brain region. We combined the drug-set analysis results across brain regions by meta-analyses of the respective p-values. We employed two different algorithms, Fisher's method ${ }^{37}$ and Tippett's minimum $p$ approach $^{38}$ to combine the p-values. Analyses were performed with the R package "metap". Multiple testing correction was 
performed by the false discovery rate (FDR) approach.

\section{RESULTS}

The sample sizes of the GWAS datasets we used are listed in Supplementary Table 1. The top 15 repositioned drug candidates for each disorder and brain region (with manual curations of drug descriptions and potential therapeutic relationship to the disorder) are presented in full in Supplementary Tables 2-9. Selected drug candidates within the top lists are presented and discussed below.

\section{Drug candidates repositioned for different psychiatric disorders}

\section{Schizophrenia}

Table 1 shows selected candidates for schizophrenia and bipolar disorder. For schizophrenia, it is interesting to note that we identified a number of known antipsychotics such as thioproperazine, droperidol, triflupromazine, thiethylperazine, spiperone and pimozide as top candidates. It is worth noting that our repositioning method is blind to any knowledge about existing psychiatric drugs or known drug targets. The results provide further evidence to the role of the dopaminergic system in the treatment of schizophrenia. Some top candidates have been shown to improve negative symptoms in clinical studies, such as the $\mathrm{H}_{2}$ antagonist ranitidine ${ }^{39}$ and the alpha 2 receptor antagonist idazoxan ${ }^{40}$. The antidepressant paroxetine was also tested in a double-blind clinical trial and shown to be efficacious in ameliorating negative symptoms in schizophrenia ${ }^{41}$. There are other drug candidate with preliminary support by preclinical or clinical studies with diverse mechanisms, such as the serotonin and dopamine antagonist metitepine ${ }^{42}$, the Na-K-Cl cotransporter 1 inhibitor bumetanide ${ }^{43}$ and the non-steroidal anti-inflammatory drug (NSAID) meclofenamic acid ${ }^{44}$. It is also noteworthy that a relatively large proportion of drugs with stronger literature support were derived from comparison with expressions in the frontal cortex, a brain region strongly implicated in 
schizophrenia $^{45}$.

\section{Bipolar disorder}

As for bipolar disorder, we found a number of antipsychotics among the top list (Table 2). Antipsychotics are well-known to be effective for BD overall and for the associated psychotic symptoms. Our analyses also revealed other candidates with known or potential antidepressant effects, such as imipramine, yohimbine, metyrapone ${ }^{46}$ and ketoconazole ${ }^{47}$. The latter two drugs are believed to exert antidepressant effects by reducing cortisol levels. Antidepressants are often used in the treatment of bipolar patients ${ }^{48}$. While there are controversies regarding its use, anti-depressants are included as valid treatment options in current guidelines, especially in bipolar II patients or when used with a mood stabilizer ${ }^{49}$. Interestingly, a few NSAIDs were also on the top list, such as aspirin and a cyclooxygenase-2 (COX-2) inhibitor SC-58125, which is supported by the neuro-inflammatory hypothesis of $\mathrm{BD}^{50}$. In line with the observation of raised cardiovascular risks in bipolar patients and possible shared pathophysiology between these disorders ${ }^{51}$, simvastatin and metformin were also among the top list.

\section{Major depressive disorder}

Two sets of GWAS data for MDD were used in our analyses (Table 3). For the results using the MDD-PGC data, we observed that fluoxetine, a widely used selective serotonin reuptake inhibitor (SSRI), was among the top candidates. We also observed quite a few antipsychotic medications on the list, such as sulpride, promazine, perphenazine and loxapine. Other repositioning candidates are more diverse in their mechanisms, such as phosphodiesterase inhibitors (papaverine ${ }^{52}$ ), histamine receptor antagonists (thioperamide ${ }^{52}$ ), muscarinic antagonists (scopolamine ${ }^{53}$ ) and cortisol-lowering agents (ketoconazole ${ }^{47}$ ) etc.

As for the repositioning results from the MDD-CONVERGE study, a known SSRI 
$\left(\right.$ zimeldine $\left.^{54}\right)$, a norepinephrine-dopamine reuptake inhibitor (nomifensine ${ }^{55}$ ) and a monoamine oxidase (MAO) inhibitor (isocarboxazid) were ranked among the top, although the former two drugs have been withdrawn due to other unrelated adverse effects. Two antipsychotics sulpride and risperidone were also on the list. Sulpride was tested in a double blind randomized controlled trial (RCT) of 177 patients and shown to significantly improve depressive symptoms ${ }^{56}$. Risperidone was confirmed to be useful as adjunctive treatment for $\mathrm{MDD}^{57}$. Interestingly, we found three drugs with actions on the glutamatergic system among the top candidates ${ }^{58}$. Arcaine and ifenprodil are NMDA antagonists and cycloserine is a partial agonist at the glycine site of the NMDA glutamate receptor. Ifenprodil was shown to improve depression in a mouse model ${ }^{59}$ while cycloserine has been shown to be effective as an add-on therapy in an $\mathrm{RCT}^{60}$.

\section{Anxiety disorders}

For anxiety disorders, we found the SSRI paroxetine and the tricyclic antidepressant protriptyline were on the top list. Another drug on the serotonergic system, pirenperone acts as a 5-HT2 antagonist and was shown anxiolytic actions in a small clinical study ${ }^{61}$. Some other drugs with preliminary support by animal or clinical studies include bumetanide, a loop diuretic which also affects $\mathrm{GABA}_{\mathrm{A}}$ signaling ${ }^{62}$; ivermectin, an anti-parasitic and GABA agonist $^{63}$; kawain, a kavalactone with anxiolytic properties shown in a number of clinical trials $^{64}$; nimodipine, a calcium channel blocker with potential anxiolytic effects ${ }^{65}$ and piracetam, a nootropic drug which is also proposed for treatment of dementia ${ }^{66}$.

\section{Alzheimer disease}

$\mathrm{AD}$ is a relatively intense research area and we have found numerous drugs with some support by preclinical or clinical studies. Some of the drugs among the top candidates received have been tested in clinical trials. Naftidrofuryl is a vasodilatory agent which was 
shown to be effective for functional outcomes, mood and cognitive function in a meta-analysis ${ }^{67}$. Vinpocetine is an alkaloid from a plant which showed some benefits in dementia according to a meta-analysis of three $\mathrm{RCTs}^{68}$. Some other interesting repositioning candidates include verapamil, a calcium channel blocker ${ }^{69}$; piribedil, a D2/D3 agonist $^{70}$; luteolin kaempferol and quercetin, flavonoids with potential anti-dementia effects ${ }^{71}$; harmine, an alkaloid which may inhibit tau phosphorylation in $\mathrm{AD}^{72}$ etc. Interestingly, quite a number of NSAIDs appeared on our top list of drugs, such as meclofenamic acid, ketorolac, celecoxib, naproxen and acemetacin. NSAIDs have been tested in clinical studies for possible prevention or treatment of $\mathrm{AD}$, although the results were inconsistent and further investigations are required $^{73}$.

\section{Attention deficit hyperactivity disorder}

A few drugs on the top list have been tested in clinical trials. The anticonvulsant carbamazepine was analyzed in a meta-analysis which concluded preliminary evidence in treating ADHD. The alkaloid lobeline may improve working memory in adult $\mathrm{ADHD}^{74}$. Tranylcypromine, another MAOI among the top list, was effective in clinical trials although its side effects need to be considered ${ }^{75}$. Other drugs such as rolipram and reserpine were found to be effective for ADHD-like symptoms in animal models ${ }^{76,77}$.

\section{Autistic spectrum disorders}

A few drugs on the top list are worth mentioning. Risperidone is one of the two FDA-approved medication for treating irritability in ASD. Two drugs, pentoxylline (phosphodiesterase inhibitor) and amantadine (NMDA antagonist), have been tested in clinical trials of ASD as combination treatment with risperidone. Both showed effectiveness in ameliorating behavioral problems ${ }^{78,79}$. Another drug loxapine, a typical anti-psychotic, was effectiveness as an add-on therapy for irritability in $\mathrm{ASD}^{80}$. Ribavirin is another interesting 
candidate. Recently autism was shown to be associated with eIF4E overexpression which in turn leads to excessive translation of neuroligins ${ }^{81}$. Ribavarin, an antiviral agent, was found to be an inhibitor of eIF4 $\mathrm{E}^{82}$ and hence may serve as a potential treatment. Ribavarin was also listed in a patent for autism treatment (www.google.ch/patents/US5008251).

\section{Drug-set enrichment analyses}

\section{Enrichment for drugs listed in the ATC classification system}

First we considered the enrichment test results from drugs listed in the ATC classification system. As shown in Table 4, antipsychotics were strongly enriched in the repositioning candidates for SCZ (lowest $p$ across four tests $=4.69 \mathrm{E}-06$ ) and $\mathrm{BD}$ (lowest $p=2.26 \mathrm{E}-07$ ). Interestingly, antipsychotics were also enriched in the drug candidates (albeit less strongly) for $\operatorname{MDD}$ (lowest $\mathrm{p}=0.0285)$, $\mathrm{AD}$ (lowest $\mathrm{p}=0.0256$ ) and anxiety disorders (lowest $\mathrm{p}=$ 0.0054). We also observed antidepressants and anxiolytics to be enriched in drugs repositioned for bipolar disorder (lowest $\mathrm{p}=1.17 \mathrm{E}-05$ ). In addition we found a trend towards significance for $\mathrm{AD}$ (lowest $\mathrm{p}=0.0507$ ). When all psychiatric medications are combined as a drug-set, evidence of enrichment was found for SCZ, BD, AD, and ANX.

\section{Enrichment for drugs listed in MEDI-HPS}

The enrichment test results using MEDI-HPS drugs were in general consistent with those derived from the ATC drugs (Table 5). Again we observed that antipsychotics were highly enriched in the repositioning candidates for schizophrenia (lowest $\mathrm{p}=2.24 \mathrm{E}-09$ ). For the rest of the analyses with the antipsychotics drug-set, we found enrichment for BD and MDD; a nominally significant result was also observed for AD.

When the drug-set was limited to antidepressants and anxiolytics, enrichment was observed for SCZ (lowest $\mathrm{p}=1.52 \mathrm{E}-03$ ), BD (lowest $\mathrm{p}=0.0143$ ) and $\mathrm{AD}$ (lowest $\mathrm{p}=0.0458$ ). When 
all psychiatric drugs were included, enrichment was found for SCZ, BD and AD.

\section{Enrichment for drugs listed in clinicalTrial.gov}

We tested for enrichment for drugs listed in clinicalTrial.gov for each of the corresponding disorders (Table 6). (We did not pursue tests of drugs across diagnoses as drugs listed in clinicalTrial.gov are less certain and well-defined as a "drug class" compared to the previous two sources). Evidence of enrichment was observed for SCZ (lowest $\mathrm{p}=0.0116$ ), BD (lowest $\mathrm{p}=0.0132)$ MDD (lowest $\mathrm{p}=0.0396)$ and anxiety disorders (lowest $\mathrm{p}=0.0066$ ).

\section{DISCUSSION}

In this study we developed a novel framework for drug repositioning by linking two apparently disparate sources of information, GWAS and the Connectivity Map. We proposed to compare the GWAS-imputed transcriptome profiles with those derived from drugs. We applied the methodology to 7 psychiatric disorders and identified a number of interesting candidates for repositioning, many of which are supported by animal studies or clinical trials. The drug-set enrichment analyses further lend support to the validity of our approach.

There are a number of advantages of our repositioning framework. Firstly this approach is largely "hypothesis-free" in the manner that it does not assume any knowledge about known drug targets or drug-disease relationships. As a result the method may be able to find drugs of different mechanisms from the known treatments. This method may be particularly useful for diseases with few known treatments or if existing treatments are highly similar in their mechanisms. A related advantage is that it can be applied to any chemicals as long as the expression profile is available, such as traditional Chinese medicine (TCM) products with no known drug targets ${ }^{83}$. As described before, another important advantage when compared to conventional connectivity mapping is that GWAS-imputed transcriptome is relatively immune 
to confounding by medication effects. With the availability of eQTL reference resources such as GTEx, imputation of expression profiles can be performed easily for close to a hundred tissues. In addition, only GWAS summary statistics are required as input for imputation, which obviates the difficulties in obtaining raw genotype data and makes the approach easily applicable to a wide variety of traits. The current method is also intuitive and computationally simple to implement. Moreover, we have considered all genetic variants instead of just the most significant hits in our repurposing pipeline. One alternative for inclusion of sub-threshold associations is to employ gene-set analysis to look for over-representation of genes acting as drug targets ${ }^{84}$, but here we proposed a different novel approach that does not rely on knowledge of known drug targets and takes into account the direction of genetic associations.

It is encouraging to observe that our repositioning results are in general supported by the drug-set enrichment analyses. In particular, antipsychotics, which are known to treat SCZ and $\mathrm{BD}$, are also strongly enriched in the repositioning candidates of these two disorders. Similarly, antidepressant is an indicated treatment option of $\mathrm{BD}^{49}$, which was also significantly enriched for this disorder. For MDD (using the PGC data) and anxiety disorders, while we did not observe enrichment in ATC or MEDI-HPS drug-sets, there was some evidence that our approach preferentially picked up drugs included in clinical trials. These results suggest that GWAS results contain useful information for drug discovery or repositioning, and is concordant with the conclusion of a previous study that drugs supported by human genomic data increase along the development pipeline ${ }^{7}$.

Interestingly, our analyses also revealed possible enrichment of antipsychotics for MDD, anxiety disorders and AD. Antipsychotics have long been used for treatment of depression, especially in the more severe cases with psychotic symptoms. A recent meta-analysis also 
demonstrated efficacy of a number of atypical antipsychotics as adjunctive treatment in depression $^{85}$. Given the high co-morbidity of depression and anxiety ${ }^{86}$ and possibly shared pathophysiology ${ }^{87}$, it is not unexpected that antipsychotics may be useful for anxiety as well. Anti-psychotics are not infrequently prescribed for anxiety disorders, although its safety and efficacy warrant further investigations ${ }^{88}$. In a similar vein, we observed that antidepressants and anti-anxiety agents were over-represented in the top drug lists of SCZ and AD (with nominally significance for AD). Psychotic and depressive symptoms are very commonly $\operatorname{seen}^{89}$ in $\mathrm{AD}$ patients, hence the enrichment for antipsychotics and antidepressants are expected. Antidepressants have been tested in clinical trials for schizophrenia, especially for negative symptoms, although further studies are required in this $\operatorname{area}^{90}$. Our findings provide a genetic basis for the effectiveness of psychiatric drugs across diagnoses.

We observed that for some of the psychiatric disorders there were no significant enrichment of known drug indications, or the enrichment came from another class of drugs. There are a few possible explanations. Firstly, the sample size may not be large enough to detect enrichment of known drugs. Among the eight datasets, the sample size of SCZ GWAS is the largest, reaching almost 80000 . For the other traits, the sample sizes are mostly between 5000 to 20000. Limited sample sizes imply that some true associations are not detected and the imputed transcriptome is less accurate, which will affect the ability to find drugs with matched (reversed) expression profiles. For any high-throughput studies with limited sample sizes, it is possible for some signals (here known drug indications) to be "missed", although other associations may be detected. An analogy is that earlier SCZ GWAS with smaller sample sizes did not detect the DRD2 locus but revealed other loci such as ZNF804A ${ }^{91}$. With accumulation of samples, the latest GWAS meta-analysis did confirm DRD2 as a susceptibility gene ${ }^{23}$. Hence limited sample sizes may explain, for instance, the enrichment of antipsychotics for MDD but not antidepressants. 
Secondly, many psychiatric disorders are known to be heterogeneous. For MDD, anxiety disorders and $\mathrm{AD}$, the prevalences are relatively high $^{92,93}$ and there may be a wider range of heterogeneity in clinical manifestations and the underlying pathophysiologies. The heterogeneity impairs study power and implies that a specific drug or drug class may only be effective for a certain group of patients. Third, there are limited available treatment for some disorders, especially AD, AUT and ADHD, which makes the detection of these known drug indications difficult.

In addition to the above, we observed that enrichment analyses were mostly negative for the MDD-CONVERGE dataset. One possible explanation is that as the GTEx dataset from which transcriptome imputation is based is not well-matched to the MDD-CONVERGE sample. The latter sample is composed of Chinese women, while the GTEx project includes only $\quad 1.0 \%$ Asians and $34.4 \%$ of females to date (http://www.gtexportal.org/home/tissueSummaryPage, accessed $22^{\text {nd }}$ Dec 2016). While we expect overlap in the genetics of expression regulation, the imputation quality may be affected. Notwithstanding some negative results in the drug enrichment analyses, many of the top candidates for repositioning are suggested in preclinical or clinical studies, and are still worthy of further investigations.

There are a few general limitations to our approach. It is worth noting that the GWAS-imputed transcriptome captures the genetically regulated part of expression, and expression changes due to other factors (e.g. environmental risk factors) cannot be modelled. The method of comparing expressions is largely "hypothesis-free" as mentioned previously, however it may be improved by incorporating knowledge on drug targets or drug-disease relationships, if such information is available. The drug expression profiles were not 
measured in brain tissues in Cmap, although the original publication on Cmap showed that drugs on neuropsychiatric diseases such as Alzheimer's disease or schizophrenia can still be reasonably modelled ${ }^{13}$. It should be emphasized that the best approach to verify the repositioning predictions should rest on careful and adequately-sized preclinical and clinical studies, and the current study does not provide confirmatory evidence for the repositioning candidates.

In conclusion, we have developed a novel framework for drug repositioning by linking up GWAS and drug expression profiling and applied the methodology to 7 psychiatric disorders. Our analyses also provide support that the psychiatric GWAS signals are enriched for known drug indications. We also present a list of repositioning candidates for each disorder, which will we believe will serve as a useful resource for preclinical and clinical researchers to pursue further studies.

\section{Acknowledgements}

This study is partially supported by the Lo-Kwee Seong Biomedical Research Fund and a Direct Grant from the Chinese University of Hong Kong. We would like to thank Mr. CHAN Chun Wing for assistance in the annotation of drugs. We are grateful to Professor Stephen K.W. Tsui for useful discussions and the Hong Kong Bioinformatics Centre for computing support. 
bioRxiv preprint doi: https://doi.org/10.1101/096503; this version posted December 23, 2016. The copyright holder for this preprint (which was not certified by peer review) is the author/funder. All rights reserved. No reuse allowed without permission. 
Table 1 Selected drug repositioning candidates for schizophrenia

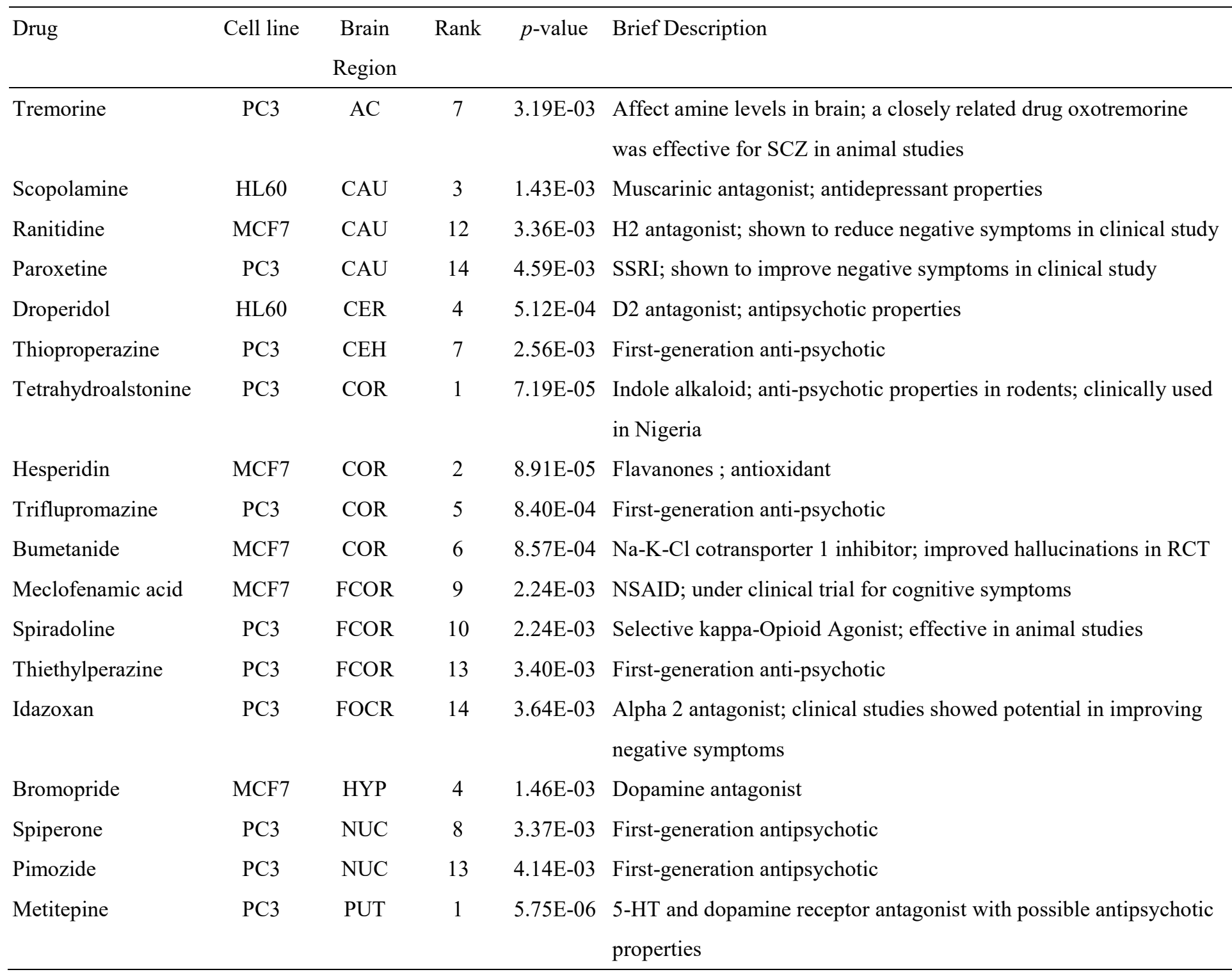

"Rank" refers to the rank within each brain region for the studied disorder. Please refer to the supplementary Table 2 for corresponding references. AC: Anterior cingulate cortex BA24; CAU: Caudate (of basal ganglia); CER: cerebellum; CEH: cerebellar hemisphere; COR: cortex; FCOR: frontal cortex (BA9); HIP, hippocampus; HYP, hypothalamus; NUC, nucleus accumbens (of basal ganglia); PUT, putamen (of basal ganglia). RCT: randomized controlled trial; NSAID, no-steroidal anti-inflammatory drugs. 
Table 2 Selected drug repositioning candidates for bipolar disorder

\begin{tabular}{|c|c|c|c|c|c|}
\hline Drug & Cell line & $\begin{array}{l}\text { Brain } \\
\text { Region }\end{array}$ & Rank & $p$-value & Brief Description \\
\hline Norcyclobenzaprine & PC3 & CAU & 1 & $3.31 \mathrm{E}-04$ & Structurally very similar to the antidepressant amitriptyline \\
\hline Acetylsalicylsalicylic acid & PC3 & CAU & 2 & $3.65 \mathrm{E}-04$ & Closely related to aspirin, which may be useful for BD \\
\hline Thioridazine & HL60 & CAU & 6 & $1.03 \mathrm{E}-03$ & First generation antipsychotic \\
\hline Mexiletine & PC3 & CER & 6 & $2.25 \mathrm{E}-03$ & $\begin{array}{l}\text { Class IB anti-arrthymic; may be useful for treatment } \\
\text { resistant BD }\end{array}$ \\
\hline Dextromethorphan & HL60 & CER & 11 & 4.23E-03 & Morphinan class; may be effective for bipolar depression \\
\hline Imipramine & HL60 & CER & 14 & $5.56 \mathrm{E}-03$ & Tricyclic antidepressant \\
\hline $\begin{array}{l}\text { Prestwick-689 } \\
\text { (androsterone) }\end{array}$ & MCF7 & COR & 13 & $2.53 \mathrm{E}-03$ & $\begin{array}{l}\text { DHEA (precursor of this drug) may have anti-depressant } \\
\text { effects }\end{array}$ \\
\hline Alpha yohimbine & MCF7 & HIP & 1 & $3.82 \mathrm{E}-04$ & $\begin{array}{l}\text { Alpha } 2 \text { antagonist; an RCT showed yohimbine hastened } \\
\text { anti-depressant response if combined with fluoxetine }\end{array}$ \\
\hline Trifluoperazine & MCF7 & HIP & 5 & $1.22 \mathrm{E}-03$ & Antipsychotic \\
\hline Molindone & MCF7 & HIP & 12 & $3.72 \mathrm{E}-03$ & Antipsychotic \\
\hline Metformin & MCF7 & HIP & 13 & $3.90 \mathrm{E}-03$ & Antidiabetic; being tested in clinical trial for refractory BD \\
\hline Thioproperazine & MCF7 & HYP & 10 & $3.54 \mathrm{E}-03$ & First generation antipsychotic \\
\hline Mesoridazine & PC3 & NUC & 6 & $1.99 \mathrm{E}-03$ & First generation antipsychotic \\
\hline Metitepine & PC3 & PUT & 1 & $5.75 \mathrm{E}-06$ & $\begin{array}{l}\text { 5-HT and dopamine receptor antagonist with possible } \\
\text { antipsychotic properties }\end{array}$ \\
\hline
\end{tabular}

\footnotetext{
"Rank" refers to the rank within each brain region for the studied disorder. Please refer to the supplementary Table 3 for corresponding references. Please refer to Table 1 for the abbreviations of brain regions. RCT: randomized controlled trial; BD, bipolar disorder; MDD, major depressive disorder.
} 
Table 3 Selected drug repositioning candidates for major depressive disorder

\begin{tabular}{|c|c|c|c|c|c|}
\hline Drug & Cell line & $\begin{array}{l}\text { Brain } \\
\text { Region }\end{array}$ & Rank & p-value & Brief Description \\
\hline \multicolumn{6}{|l|}{ MDD-PGC } \\
\hline Papaverine & HL60 & $\mathrm{AC}$ & 3 & $2.93 \mathrm{E}-04$ & $\begin{array}{l}\text { Cyclic nucleotide phosphodiesterase inhibitor; shown to be useful for depression } \\
\text { in a case report }\end{array}$ \\
\hline Fluoxetine & MCF7 & CAU & 3 & $6.87 \mathrm{E}-04$ & SSRI antidepressant \\
\hline Sulpiride & PC3 & CAU & 12 & $4.46 \mathrm{E}-03$ & Antipsychotic with potential antidepressant properties \\
\hline Promazine & MCF7 & CAU & 15 & 4.89E-03 & Antipsychotic with potential antidepressant properties \\
\hline Sanguinarine & HL60 & CER & 1 & $3.59 \mathrm{E}-04$ & $\begin{array}{l}\text { A selective mitogen-activated protein kinase phosphatase-1 (Mkp-1) } \\
\text { inhibitor; showed antidepressant effects in rats }\end{array}$ \\
\hline Thioperamide & MCF7 & CER & 4 & $8.80 \mathrm{E}-04$ & Histamine $\mathrm{H} 3$ and $\mathrm{H} 4$ receptor antagonist; antidepressant effects in rats \\
\hline Pregnenolone & PC3 & $\mathrm{CEH}$ & 4 & $1.09 \mathrm{E}-03$ & Endogenous steroid; shown to be useful in bipolar depression in RCT \\
\hline Scopolamine & HL60 & $\mathrm{CEH}$ & 13 & $4.44 \mathrm{E}-03$ & Anti-muscarinic agent which showed antidepressant effects in at least 2 RCTs \\
\hline Vitexin & PC3 & COR & 7 & $2.00 \mathrm{E}-03$ & Flavone glucoside; antidepressant properties shown in mice \\
\hline Pirlindole & MCF7 & FCOR & 9 & $2.85 \mathrm{E}-03$ & A reversible inhibitor of monoamine oxidase (MAO) A \\
\hline Palmatine & HL60 & HIP & 10 & $2.06 \mathrm{E}-03$ & An alkaloid in plants, shown to have antidepressant properties in mice \\
\hline Perphenazine & MCF7 & HYP & 1 & $2.59 \mathrm{E}-05$ & Typical antipsychotic with possible antidepressant properties \\
\hline Loxapine & HL60 & HYP & 7 & $1.94 \mathrm{E}-03$ & Typical antipsychotic with possible antidepressant properties \\
\hline Pyridoxine & PC3 & NUC & 5 & $1.07 \mathrm{E}-03$ & Vitamin B6; may have antidepressant effects in pre-menopausal women \\
\hline Ketoconazole & PC3 & NUC & 14 & $2.75 \mathrm{E}-03$ & $\begin{array}{l}\text { Antidepressant properties suggested in case series, possibly via } \\
\text { cortisol-lowering effects }\end{array}$ \\
\hline Piroxicam & HL60 & PUT & 10 & $2.46 \mathrm{E}-03$ & NSAID with possible antidepressant actions shown in mice \\
\hline
\end{tabular}

\section{MDD-CONVERGE}

$\begin{array}{lll}\text { Kawain } & \text { PC3 } & \text { AC } \\ \text { Doxycycline } & \text { PC3 } & \text { AC } \\ \text { Idazoxan } & \text { PC3 } & \text { CAU } \\ \text { Nomifensine } & \text { HL60 } & \text { CER } \\ \text { Arcaine } & \text { HL60 } & \text { COR } \\ & & \\ \text { Zimeldine } & \text { PC3 } & \text { COR } \\ \text { Bromocriptine } & \text { PC3 } & \text { FCOR } \\ \text { Isocarboxazid } & \text { HL60 } & \text { FOCR } \\ \text { Pioglitazone } & \text { MCF7 } & \text { FOCR } \\ \text { Sulpiride } & \text { HL60 } & \text { HIP } \\ \text { Risperidone } & \text { HL60 } & \text { HYP } \\ \text { Cycloserine } & \text { HL60 } & \text { PUT } \\ \text { Ifenprodil } & \text { HL60 } & \text { PUT }\end{array}$

6.90E-04 Effective for treatment of anxiety shown in RCTs

1.79E-03 Antidepressant properties shown in mice

1.10E-03 Alpha 2 antagonist; clinical studies showed effects in bipolar depression

$1.56 \mathrm{E}-03$ A norepinephrine-dopamine reuptake inhibitor, anti-depressant

3.91E-04 NMDA antagonist; this drug class was shown to produce antidepressant effects in animal studies and RCT

1.94E-03 SSRI antidepressant

3.16E-05 Dopamine agonist; antidepressant effects in a small open study

1.00E-03 MAO inhibitor

2.95E-03 Antidepressant properties when combined with citalopram in a RCT

2.34E-03 Antipsychotic with potential antidepressant properties

2.25E-03 Atypical antipsychotic useful for augmentation as shown in RCT

9.86E-04 Acts on NMDA receptor; clinical trial showed benefits in MDD

1.18E-03 NMDA antagonist; found to potentiate the effects of SSRI and TCA in mice

"Rank" refers to the rank within each brain region for the studied disorder. Please refer to the supplementary Table 4-5 for corresponding 
bioRxiv preprint doi: https://doi.org/10.1101/096503; this version posted December 23, 2016. The copyright holder for this preprint (which was not certified by peer review) is the author/funder. All rights reserved. No reuse allowed without permission.

references. Please refer to Table 1 for the abbreviations of brain regions. RCT: randomized controlled trial; SSRI, selective serotonin reuptake inhibitor; TCA, tricyclic antidepressant. 
Table 4 Drug enrichment analyses with drug-sets defined in the Anatomical Therapeutic Chemical (ATC) Classification System

\begin{tabular}{|c|c|c|c|c|}
\hline Disorder & Self (fisher) & Compet (fisher) & Self (minp) & Compet (minp) \\
\hline \multicolumn{5}{|c|}{ Enrichment for ATC antipsychotics } \\
\hline $\mathrm{SCZ}$ & $6.78 E-06$ & 4.69E-06 & 0.0011 & 0.0010 \\
\hline $\mathrm{BD}$ & 4.14E-07 & 2.26E-07 & 0.0031 & 0.0025 \\
\hline MDD & 0.1634 & 0.1532 & 0.0322 & 0.0285 \\
\hline MDD-CONVERGE & 0.4484 & 0.4231 & 0.6674 & 0.6546 \\
\hline $\mathrm{AD}$ & 0.6849 & 0.6684 & 0.0294 & 0.0256 \\
\hline ANX & 0.0249 & 0.0241 & 0.0058 & 0.0054 \\
\hline ADHD & 0.9215 & 0.9209 & 0.7887 & 0.7939 \\
\hline AUT & 0.9956 & 0.9949 & 0.3816 & 0.3523 \\
\hline
\end{tabular}

Enrichment for ATC antidepressants or anxiolytics

$\begin{array}{lcccc}\text { SCZ } & 0.1141 & 0.1075 & 0.1301 & 0.1264 \\ \text { BD } & \mathbf{1 . 6 4 E - 0 5} & \mathbf{1 . 1 7 E - 0 5} & \mathbf{0 . 0 0 5 5} & \mathbf{0 . 0 0 5 0} \\ \text { MDD } & 0.9911 & 0.9908 & 0.9448 & 0.9420 \\ \text { MDD-CONVERGE } & 0.7199 & 0.7016 & 0.3904 & 0.3693 \\ \text { AD } & 0.3475 & 0.3323 & 0.0564 & 0.0507 \\ \text { ANX } & 0.1941 & 0.1953 & 0.2248 & 0.2173 \\ \text { ADHD } & 0.9904 & 0.9904 & 0.9654 & 0.9641 \\ \text { AUT } & 0.8343 & 0.8400 & 0.7034 & 0.6884\end{array}$

Enrichment for all ATC psychiatric drugs

\begin{tabular}{lcccc} 
SCZ & $\mathbf{8 . 2 0 E - 0 5}$ & $\mathbf{4 . 2 2 E - 0 5}$ & $\mathbf{0 . 0 1 6 0}$ & $\mathbf{0 . 0 1 3 0}$ \\
BD & $\mathbf{5 . 7 0 E - 0 9}$ & $\mathbf{1 . 2 0 E - 0 9}$ & $\mathbf{0 . 0 0 3 3}$ & $\mathbf{0 . 0 0 2 2}$ \\
MDD & 0.7234 & 0.6935 & 0.1738 & 0.1482 \\
MDD-CONVERGE & 0.6548 & 0.6130 & 0.6718 & 0.6329 \\
AD & 0.1695 & 0.1360 & $\mathbf{0 . 0 0 2 2}$ & $\mathbf{0 . 0 0 1 3}$ \\
ANX & $\mathbf{0 . 0 0 6 1}$ & $\mathbf{0 . 0 0 4 8}$ & $\mathbf{0 . 0 1 2 9}$ & $\mathbf{0 . 0 1 1 0}$ \\
ADHD & 0.9981 & 0.9982 & 0.8358 & 0.8367 \\
AUT & 0.9988 & 0.9988 & 0.9542 & 0.9406 \\
\hline
\end{tabular}

Self (fisher): self-contained test (one-sample $t$-test) combined across brain regions by Fisher's method; Compet (fisher), competitive test (two-sample $t$-test) combined across brain regions by Fisher's method; Self (minp), self-contained test (one-sample $t$-test) combined across brain regions by Tippett's minimum $p$ method; Compet (minp), competitive test (two-sample $t$-test) combined across brain regions by Tippett's minimum $p$ method. Test results with $p<0.05$ and $q$-value $<0.2$ are in bold. Full tables of $q$-values are presented in Supplementary Table 10-12.

SCZ, schizophrenia; BD, bipolar disorder; MDD, major depressive disorder with GWAS data from the Psychiatric Genomics Consortium; MDD-CONVERGE, major depressive disorder with GWAS data from the CONVERGE Consortium; AD, Alzheimer's disease; ANX, anxiety disorders; ADHD, attention deficit hyperactivity disorder; $\mathrm{AU}$, autistic spectrum disorders. 

was not certified by peer review) is the author/funder. All rights reserved. No reuse allowed without permission.

Table 5 Drug enrichment analyses with drug-sets defined by MEDI-HPS

\begin{tabular}{|c|c|c|c|c|}
\hline Disorder & Self (fisher) & $\begin{array}{l}\text { Compet } \\
\text { (fisher) }\end{array}$ & Self (minp) & $\begin{array}{l}\text { Compet } \\
\text { (minp) }\end{array}$ \\
\hline
\end{tabular}

Enrichment for indicated drugs of each disorder

$\begin{array}{lcccc}\text { SCZ } & \mathbf{4 . 4 6 E - 0 9} & \mathbf{2 . 2 4 E - 0 9} & \mathbf{1 . 9 7 E - 0 2} & \mathbf{1 . 7 0 E - 0 2} \\ \text { BD } & 0.9962 & 0.9960 & 0.9982 & 0.9982 \\ \text { MDD } & 0.4724 & 0.4545 & 0.4525 & 0.4210 \\ \text { MDD-CONVERGE } & 0.5120 & 0.4787 & 0.7166 & 0.6945 \\ \text { AD } & 0.5686 & 0.5646 & 0.1148 & 0.1140 \\ \text { ANX } & 0.7188 & 0.7224 & 0.6363 & 0.6195 \\ \text { ADHD } & 0.6374 & 0.6355 & 0.4404 & 0.4359 \\ \text { AUT } & \text { NA } & \text { NA } & \text { NA } & \text { NA }\end{array}$

Enrichment for antispsychotics

$\begin{array}{lcccc}\text { SCZ } & \mathbf{4 . 4 6 E - 0 9} & \mathbf{2 . 2 4 E - 0 9} & \mathbf{0 . 0 1 9 7} & \mathbf{0 . 0 1 7 0} \\ \text { BD } & \mathbf{5 . 8 2 E - 0 3} & \mathbf{4 . 4 6 E - 0 3} & 0.0950 & 0.0890 \\ \text { MDD } & 0.0850 & 0.0795 & \mathbf{0 . 0 2 9 3} & \mathbf{0 . 0 2 9 0} \\ \text { MDD-CONVERGE } & 0.4266 & 0.3933 & 0.2645 & 0.2321 \\ \text { AD } & 0.3318 & 0.3096 & 0.0546 & \mathbf{0 . 0 4 7 9} \\ \text { ANX } & 0.2963 & 0.2949 & 0.2099 & 0.2061 \\ \text { ADHD } & 0.6671 & 0.6578 & 0.2597 & 0.2419 \\ \text { AUT } & 0.9311 & 0.9235 & 0.1326 & 0.1149\end{array}$

Enrichment for antidepressants or anxiolytics

$\begin{array}{lcccc}\text { SCZ } & \mathbf{1 . 9 2 E - 0 3} & \mathbf{1 . 5 2 E - 0 3} & \mathbf{0 . 0 3 1 6} & \mathbf{0 . 0 2 8 2} \\ \text { BD } & \mathbf{0 . 0 1 7 3} & \mathbf{0 . 0 1 4 3} & \mathbf{0 . 0 2 1 4} & \mathbf{0 . 0 1 8 9} \\ \text { MDD } & 0.4724 & 0.4545 & 0.4525 & 0.4210 \\ \text { MDD-CONVERGE } & 0.5120 & 0.4787 & 0.7166 & 0.6945 \\ \text { AD } & 0.3293 & 0.3104 & 0.0530 & \mathbf{0 . 0 4 5 8} \\ \text { ANX } & 0.7188 & 0.7224 & 0.6363 & 0.6195 \\ \text { ADHD } & 0.9975 & 0.9975 & 0.7589 & 0.7435 \\ \text { AUT } & 0.9880 & 0.9879 & 0.8248 & 0.8112\end{array}$

Enrichment for all psychiatric drugs

$\begin{array}{lcccc}\text { SCZ } & \mathbf{1 . 2 1 E - 0 7} & \mathbf{4 . 4 1 E - 0 8} & \mathbf{1 . 0 2 E - 0 3} & \mathbf{7 . 4 4 E - 0 4} \\ \text { BD } & \mathbf{7 . 6 7 E - 0 4} & \mathbf{4 . 1 3 E - 0 4} & \mathbf{6 . 5 9 E - 0 3} & \mathbf{5 . 0 3 E - 0 3} \\ \text { MDD } & 0.2691 & 0.2471 & 0.2859 & 0.2878 \\ \text { MDD-CONVERGE } & 0.5842 & 0.5316 & 0.2983 & 0.2422 \\ \text { AD } & 0.2307 & 0.1921 & \mathbf{0 . 0 1 1 7} & \mathbf{8 . 1 4 E - 0 3} \\ \text { ANX } & 0.5701 & 0.5650 & 0.4802 & 0.4488\end{array}$



was not certified by peer review) is the author/funder. All rights reserved. No reuse allowed without permission.

\begin{tabular}{lllll} 
ADHD & 0.9482 & 0.9455 & 0.6337 & 0.5999 \\
AUT & 0.9778 & 0.9738 & 0.5533 & 0.4971 \\
\hline
\end{tabular}

Please refer to Table 4 for abbreviations. Test results with $p<0.05$ and $q$-value $<0.2$ are in bold. For AUT, results are not presented as there are only two matched instances.

Table 6 Drug enrichment analyses with drug-sets defined by those listed in clinicalTrial.gov

\begin{tabular}{lcccc}
\hline Disorder & $\begin{array}{c}\text { Self } \\
\text { (fisher) }\end{array}$ & $\begin{array}{c}\text { Compet } \\
\text { (fisher) }\end{array}$ & $\begin{array}{c}\text { Self } \\
\text { (minp) }\end{array}$ & $\begin{array}{c}\text { Compet } \\
\text { (minp) }\end{array}$ \\
\hline SCZ & $\mathbf{0 . 0 1 6 2}$ & $\mathbf{0 . 0 1 1 6}$ & 0.5155 & 0.4949 \\
BD & $\mathbf{0 . 0 1 6 7}$ & $\mathbf{0 . 0 1 3 2}$ & $\mathbf{0 . 0 1 7 8}$ & $\mathbf{0 . 0 1 5 8}$ \\
MDD & $\mathbf{0 . 0 4 4 8}$ & $\mathbf{0 . 0 3 9 6}$ & 0.1085 & 0.1068 \\
MDD-CONVERGE & 0.5465 & 0.4978 & 0.6540 & 0.5910 \\
AD & 0.4371 & 0.4159 & 0.4969 & 0.4642 \\
ANX & 0.0894 & 0.0783 & $\mathbf{0 . 0 1 0 0}$ & $\mathbf{0 . 0 0 6 6}$ \\
ADHD & 0.1765 & 0.1728 & 0.2834 & 0.2691 \\
AUT & 0.9502 & 0.9555 & 0.8904 & 0.9004 \\
\hline
\end{tabular}

Please refer to Table 4 for abbreviations. Test results with $p<0.05$ and $q$-value $<0.2$ are in bold. 
References:

1. Whiteford, H.A. et al. Global burden of disease attributable to mental and substance use disorders: findings from the Global Burden of Disease Study 2010. Lancet 382, 1575-86 (2013).

2. Hyman, S.E. Psychiatric drug development: diagnosing a crisis. Cerebrum 2013, 5 (2013).

3. Dudley, J.T., Deshpande, T. \& Butte, A.J. Exploiting drug-disease relationships for computational drug repositioning. Brief Bioinform 12, 303-11 (2011).

4. Sanseau, P. et al. Use of genome-wide association studies for drug repositioning. Nat Biotechnol 30, 317-20 (2012).

5. Cao, C. \& Moult, J. GWAS and drug targets. BMC Genomics 15 Suppl 4, S5 (2014).

6. Lencz, T. \& Malhotra, A.K. Targeting the schizophrenia genome: a fast track strategy from GWAS to clinic. Mol Psychiatry 20, 820-6 (2015).

7. Nelson, M.R. et al. The support of human genetic evidence for approved drug indications. Nat Genet $47,856-60$ (2015).

8. Edwards, S.L., Beesley, J., French, J.D. \& Dunning, A.M. Beyond GWASs: illuminating the dark road from association to function. Am J Hum Genet 93, 779-97 (2013).

9. Talevi, A. Multi-target pharmacology: possibilities and limitations of the "skeleton key approach" from a medicinal chemist perspective. Front Pharmacol 6, 205 (2015).

10. Wray, N.R. et al. Research review: Polygenic methods and their application to psychiatric traits. J Child Psychol Psychiatry 55, 1068-87 (2014).

11. Sirota, M. et al. Discovery and preclinical validation of drug indications using compendia of public gene expression data. Sci Transl Med 3, 96 ra77 (2011).

12. Dudley, J.T. et al. Computational repositioning of the anticonvulsant topiramate for inflammatory bowel disease. Sci Transl Med 3, 96 ra76 (2011).

13. Lamb, J. et al. The Connectivity Map: using gene-expression signatures to connect small molecules, genes, and disease. Science 313, 1929-35 (2006).

14. Jin, L. et al. Drug-repurposing identified the combination of Trolox $C$ and Cytisine for the treatment of type 2 diabetes. J Transl Med 12, 153 (2014).

15. Ung, M.H. et al. Integrated Drug Expression Analysis for leukemia: an integrated in silico and in vivo approach to drug discovery. Pharmacogenomics $J$ (2016).

16. Raghavan, R. et al. Drug discovery using clinical outcome-based Connectivity Mapping: application to ovarian cancer. BMC Genomics 17, 811 (2016).

17. Turck, C. \& SpringerLink (Online service). Biomarkers for Psychiatric Disorders. (Springer-Verlag US,, Boston, MA, 2009).

18. Gamazon, E.R. et al. A gene-based association method for mapping traits using reference transcriptome data. Nat Genet 47, 1091-8 (2015).

19. Barbeira, A. et al. MetaXcan: Summary Statistics Based Gene-Level Association Method Infers Accurate PrediXcan Results. bioRxiv, 045260 (2016).

20. Gusev, A. et al. Integrative approaches for large-scale transcriptome-wide association studies. Nat Genet 48, 245-52 (2016).

21. Consortium, G.T. The Genotype-Tissue Expression (GTEx) project. Nat Genet 45, 580-5 (2013). 
22. Battle, A. et al. Characterizing the genetic basis of transcriptome diversity through RNA-sequencing of 922 individuals. Genome Res 24, 14-24 (2014).

23. Schizophrenia Working Group of the Psychiatric Genomics, C. Biological insights from 108 schizophrenia-associated genetic loci. Nature 511, 421-7 (2014).

24. Major Depressive Disorder Working Group of the Psychiatric, G.C. et al. A mega-analysis of genome-wide association studies for major depressive disorder. Mol Psychiatry 18, 497-511 (2013).

25. consortium, C. Sparse whole-genome sequencing identifies two loci for major depressive disorder. Nature 523, 588-91 (2015).

26. Psychiatric, G.C.B.D.W.G. Large-scale genome-wide association analysis of bipolar disorder identifies a new susceptibility locus near ODZ4. Nat Genet 43, 977-83 (2011).

27. Lambert, J.C. et al. Meta-analysis of 74,046 individuals identifies 11 new susceptibility loci for Alzheimer's disease. Nat Genet 45, 1452-8 (2013).

28. Otowa, T. et al. Meta-analysis of genome-wide association studies of anxiety disorders. Mol Psychiatry 21, 1485 (2016).

29. Autism Spectrum Disorder Working Group of the Psychiatry Genomics Consortium. Dataset: PGC-ASD summary statistics from a meta-analysis of 5,305 ASD-diagnosed cases and 5,305 pseudocontrols of European descent (based on similarity to CEPH reference genotypes) (March 2015). (available at:

http://www.med.unc.edu/pgc/results-anddownloads).

30. Neale, B.M. et al. Meta-analysis of genome-wide association studies of attention-deficit/hyperactivity disorder. J Am Acad Child Adolesc Psychiatry 49, 884-97 (2010).

31. Ritchie, M.E. et al. limma powers differential expression analyses for RNA-sequencing and microarray studies. Nucleic Acids Res 43, e47 (2015).

32. Cheng, J., Yang, L., Kumar, V. \& Agarwal, P. Systematic evaluation of connectivity map for disease indications. Genome Med 6, 540 (2014).

33. de Leeuw, C.A., Mooij, J.M., Heskes, T. \& Posthuma, D. MAGMA: Generalized Gene-Set Analysis of GWAS Data. Plos Computational Biology 11(2015).

34. de Leeuw, C.A., Neale, B.M., Heskes, T. \& Posthuma, D. The statistical properties of gene-set analysis. Nat Rev Genet 17, 353-64 (2016).

35. Kanba, S. Although antidepressants and anxiolytics are frequently used together to treat depression in the acute phase, how effective is the concomitant use of these drugs? J Psychiatry Neurosci 29, 485 (2004).

36. Wei, W.Q. et al. Development and evaluation of an ensemble resource linking medications to their indications. $J$ Am Med Inform Assoc 20, 954-61 (2013).

37. Fisher, R.A. Statistical methods for research workers, ix p., 1 I., (Oliver and Boyd, Edinburgh, London,, 1925).

38. Tippett, L.H.C. The methods of statistics; an introduction mainly for workers in the biological sciences, $222 \mathrm{p}$. (Williams \& Norgate Itd., London,, 1931).

39. Mehta, V.S. \& Ram, D. Role of ranitidine in negative symptoms of schizophrenia--an open label study. Asian J Psychiatr 12, 150-4 (2014).

40. Litman, R.E., Su, T.P., Potter, W.Z., Hong, W.W. \& Pickar, D. Idazoxan and response to typical neuroleptics in treatment-resistant schizophrenia. Comparison with the atypical neuroleptic, clozapine. Br J Psychiatry 168, 571-9 (1996).

41. Jockers-Scherubl, M.C. et al. Negative symptoms of schizophrenia are improved by the addition of paroxetine to 
neuroleptics: a double-blind placebo-controlled study. Int Clin Psychopharmacol 20, 27-31 (2005).

42. Foye, W.O., Lemke, T.L. \& Williams, D.A. Foye's principles of medicinal chemistry, xviii, 1500 p. (some color) (Wolters Kluwer Health/Lippincott Williams \& Wilkins, Philadelphia, 2013).

43. Rahmanzadeh, R. et al. Effect of bumetanide, a selective NKCC1 inhibitor, on hallucinations of schizophrenic patients; a double-blind randomized clinical trial. Schizophr Res (2016).

44. Babatope T, P.R., Kahlon RS, Mohite S, Makanjoula T, Goddu S, Aimienwanu O, Venigalla H, Malouta M, Okusaga OO. Meclofenamic Acid Reduced Plasma C- Reactive Protein and Improved Scores on the NIH Toolbox Cognitive Test Battery in a Patient with Schizoaffective Disorder. Biological Psychiatry 79(2016).

45. Harrison, P.J. The neuropathology of schizophrenia. A critical review of the data and their interpretation. Brain 122 ( Pt 4), 593-624 (1999).

46. Sigalas, P.D., Garg, H., Watson, S., McAllister-Williams, R.H. \& Ferrier, I.N. Metyrapone in treatment-resistant depression. Ther Adv Psychopharmacol 2, 139-49 (2012).

47. Brown, E.S., Bobadilla, L. \& Rush, A.J. Ketoconazole in bipolar patients with depressive symptoms: a case series and literature review. Bipolar Disord 3, 23-9 (2001).

48. Cascade, E.F., Reites, J., Kalali, A.H. \& Ghaemi, N. Antidepressants in bipolar disorder. Psychiatry (Edgmont) 4, 56-8 (2007).

49. Pacchiarotti, I. et al. The International Society for Bipolar Disorders (ISBD) task force report on antidepressant use in bipolar disorders. Am J Psychiatry 170, 1249-62 (2013).

50. Muneer, A. Bipolar Disorder: Role of Inflammation and the Development of Disease Biomarkers. Psychiatry Investig 13, 18-33 (2016).

51. So, H.-C. \& Sham, P.C. Polygenic score analyses of schizophrenia and bipolar disorder with cardiometabolic traits. arXiv preprint arXiv:1611.03191 (2016).

52. Malison, R.T., Price, L.H., Nestler, E.J., Heninger, G.R. \& Duman, R.S. Efficacy of papaverine addition for treatment-refractory major depression. Am J Psychiatry 154, 579-80 (1997).

53. Furey, M.L. \& Drevets, W.C. Antidepressant efficacy of the antimuscarinic drug scopolamine: a randomized, placebo-controlled clinical trial. Arch Gen Psychiatry 63, 1121-9 (2006).

54. Norman, T.R. et al. Zimelidine: a placebo-controlled trial in depression. Psychiatry Res 8, 95-103 (1983).

55. Forrest, A., Hewett, A. \& Nicholson, P. Controlled randomized group comparison of nomifensine and imipramine in depressive illness. Br J Clin Pharmacol 4Suppl 2, 215S-220S (1977).

56. Ruther, E. et al. Antidepressant action of sulpiride. Results of a placebo-controlled double-blind trial. Pharmacopsychiatry 32, 127-35 (1999).

57. Spielmans, G.I. et al. Adjunctive atypical antipsychotic treatment for major depressive disorder: a meta-analysis of depression, quality of life, and safety outcomes. PLoS Med 10, e1001403 (2013).

58. Dang, Y.H. et al. Targeting of NMDA receptors in the treatment of major depression. Curr Pharm Des 20, 5151-9 (2014).

59. Poleszak, E. et al. Effects of ifenprodil on the antidepressant-like activity of NMDA ligands in the forced swim test in mice. Prog Neuropsychopharmacol Biol Psychiatry 46, 29-35 (2013).

60. Heresco-Levy, U. et al. A randomized add-on trial of high-dose D-cycloserine for treatment-resistant depression. Int J Neuropsychopharmacol 16, 501-6 (2013).

61. Ansseau, M., Doumont, A., Thiry, D. \& Gelders, Y. Pilot study of a specific serotonergic antagonist, pirenperone, in the treatment of anxiety disorders. Acta Psychiatr Belg 83, 517-24 (1983). 
62. Krystal, A.D., Sutherland, J. \& Hochman, D.W. Loop diuretics have anxiolytic effects in rat models of conditioned anxiety. PLoS One 7, e35417 (2012).

63. Spinosa Hde, S., Stilck, S.R. \& Bernardi, M.M. Possible anxiolytic effects of ivermectin in rats. Vet Res Commun 26, 309-21 (2002).

64. Pittler, M.H. \& Ernst, E. Efficacy of kava extract for treating anxiety: systematic review and meta-analysis. J Clin Psychopharmacol 20, 84-9 (2000).

65. Tanwani, H., Nyati, P., Atal, S. \& Churihar, R. EVALUATION OF ANTIANXIETY, ANTIDEPRESSANT AND SEDATIVE EFFECTS OF NIMODIPINE IN SWISS ALBINO MICE. International Journal of Pharmacy and Pharmaceutical Sciences 8, 260-263 (2016).

66. File, S.E. \& Hyde, J.R. Evidence that piracetam has an anxiolytic action. J Affect Disord 1, 227-35 (1979).

67. Lu, D., Song, H., Hao, Z., Wu, T. \& McCleery, J. Naftidrofuryl for dementia. Cochrane Database Syst Rev, CD002955 (2011).

68. Szatmari, S.Z. \& Whitehouse, P.J. Vinpocetine for cognitive impairment and dementia. Cochrane Database Syst Rev, CD003119 (2003).

69. Popovic, M., Caballero-Bleda, M., Popovic, N., Bokonjic, D. \& Dobric, S. Neuroprotective effect of chronic verapamil treatment on cognitive and noncognitive deficits in an experimental Alzheimer's disease in rats. Int J Neurosci 92, 79-93 (1997).

70. Nagaraja, D. \& Jayashree, S. Randomized study of the dopamine receptor agonist piribedil in the treatment of mild cognitive impairment. Am J Psychiatry 158, 1517-9 (2001).

71. Vauzour, D., Vafeiadou, K., Rodriguez-Mateos, A., Rendeiro, C. \& Spencer, J.P. The neuroprotective potential of flavonoids: a multiplicity of effects. Genes Nutr 3, 115-26 (2008).

72. Frost, D. et al. beta-carboline compounds, including harmine, inhibit DYRK1A and tau phosphorylation at multiple Alzheimer's disease-related sites. PLoS One 6, e19264 (2011).

73. Imbimbo, B.P., Solfrizzi, V. \& Panza, F. Are NSAIDs useful to treat Alzheimer's disease or mild cognitive impairment? Front Aging Neurosci 2(2010).

74. Martin, C.A. et al. Lobeline Effects on Cognitive Performance in Adult ADHD. J Atten Disord (2013).

75. Zametkin, A., Rapoport, J.L., Murphy, D.L., Linnoila, M. \& Ismond, D. Treatment of hyperactive children with monoamine oxidase inhibitors. I. Clinical efficacy. Arch Gen Psychiatry 42, 962-6 (1985).

76. Rutten, K., Basile, J.L., Prickaerts, J., Blokland, A. \& Vivian, J.A. Selective PDE inhibitors rolipram and sildenafil improve object retrieval performance in adult cynomolgus macaques. Psychopharmacology (Berl) 196, 643-8 (2008).

77. Hofer, M.A. Effects of reserpine and amphetamine on the development of hyperactivity in maternally deprived rat pups. Psychosom Med 42, 513-20 (1980).

78. Akhondzadeh, S. et al. Double-blind placebo-controlled trial of pentoxifylline added to risperidone: effects on aberrant behavior in children with autism. Prog Neuropsychopharmacol Biol Psychiatry 34, 32-6 (2010).

79. Mohammadi, M.R. et al. Double-blind, placebo-controlled trial of risperidone plus amantadine in children with autism: a 10-week randomized study. Clin Neuropharmacol 36, 179-84 (2013).

80. Hellings, J.A. et al. Loxapine add-on for adolescents and adults with autism spectrum disorders and irritability. J Child Adolesc Psychopharmacol 25, 150-9 (2015).

81. Gkogkas, C.G. et al. Autism-related deficits via dysregulated elF4E-dependent translational control. Nature 493, 371-7 (2013). 
82. Kentsis, A., Topisirovic, I., Culjkovic, B., Shao, L. \& Borden, K.L. Ribavirin suppresses elF4E-mediated oncogenic transformation by physical mimicry of the 7-methyl guanosine mRNA cap. Proc Natl Acad Sci U S A 101, 18105-10 (2004).

83. Wen, Z. et al. Discovery of molecular mechanisms of traditional Chinese medicinal formula Si-Wu-Tang using gene expression microarray and connectivity map. PLoS One 6, e18278 (2011).

84. de Jong, S., Vidler, L.R., Mokrab, Y., Collier, D.A. \& Breen, G. Gene-set analysis based on the pharmacological profiles of drugs to identify repurposing opportunities in schizophrenia. J Psychopharmacol 30, 826-30 (2016).

85. Wang, P. \& Si, T. Use of antipsychotics in the treatment of depressive disorders. Shanghai Arch Psychiatry 25, 134-40 (2013).

86. Johansson, R., Carlbring, P., Heedman, A., Paxling, B. \& Andersson, G. Depression, anxiety and their comorbidity in the Swedish general population: point prevalence and the effect on health-related quality of life. PeerJ 1, e98 (2013).

87. Frances, A. et al. Relationship of anxiety and depression. Psychopharmacology (Berl) 106 Suppl, S82-6 (1992).

88. Breier, A. Anxiety disorders and antipsychotic drugs: a pressing need for more research. Am J Psychiatry 168, 1012-4 (2011).

89. Cerejeira, J., Lagarto, L. \& Mukaetova-Ladinska, E.B. Behavioral and psychological symptoms of dementia. Front Neurol 3, 73 (2012).

90. Singh, S.P., Singh, V., Kar, N. \& Chan, K. Efficacy of antidepressants in treating the negative symptoms of chronic schizophrenia: meta-analysis. Br J Psychiatry 197, 174-9 (2010).

91. O'Donovan, M.C. et al. Identification of loci associated with schizophrenia by genome-wide association and follow-up. Nat Genet 40, 1053-5 (2008).

92. Qiu, C., Kivipelto, M. \& von Strauss, E. Epidemiology of Alzheimer's disease: occurrence, determinants, and strategies toward intervention. Dialogues Clin Neurosci 11, 111-28 (2009).

93. Kessler, R.C. et al. Lifetime prevalence and age-of-onset distributions of DSM-IV disorders in the National Comorbidity Survey Replication. Arch Gen Psychiatry 62, 593-602 (2005). 